\title{
COMPARISON BETWEEN THE DENSITY OF LANGERHANS' CELLS IN THE PREPUCE OF NEONATES AND ADULT MALES
}

\author{
Felix Mutale, Elliot Bufuku Kafumukache, Kaile Trevor
}

Correspondence to Dr. Felix Mutale. ${ }^{1}$ Department of Anatomy, School of Medicine, University of Zambia, Lusaka, Zambia. Email: mutalefelixmc@gmail.com

\section{ABSTRACT}

Langerhans cells (LCs) in epidermis function as sentinel antigen-presenting cells that can capture invading viruses like Herpes Simplex Virus, Varicella-zoster virus and Human Immunodeficiency Virus (HIV). This interaction between Langerhans cells and viruses is highly variable depending on the virus. Herpes Simplex induces apoptosis in LCs but HIV does not, instead it presents the HIV to T-cells in lymph nodes which in turn get infected. Langerhans cells in the prepuce are therefore, a portal of entry for HIV. Hence, world health organisation recommends male circumcision to reduce the densities of Langerhans cells. Fifteen fresh foreskins were obtained from adult males aged 18 years and above after circumcision, five had past history of ulcerative STI and five fresh foreskins were obtained from neonates. The specimens were fixed using $10 \%$ buffered formalin and transported to the histopathology laboratory where the tissues were grossed examined and embedded using paraffin wax. The formalin fixed paraffin blocks were then sectioned into 3-5um sections and then followed by immunohistochemistry staining. The primary monoclonal antibodies (anti-CD1a) targeting Langerhans cells were used and LCs counting was done. The mean Langerhans cells density in neonates was $36.6 \pm 5.273 / \mathrm{mm}^{2}$, while adults without past history of ulcerative STIs was $69.4 \pm 8.847 / \mathrm{mm} 2$ and those with history of ulcerative STIs was $88.4 \pm 7.273 / \mathrm{mm} 2$.Both age and past history of ulcerative STIs have an influence on the density of Langerhans cell on the prepuce. The neonates showed lower densities than adults. Adults with history of ulcerative STIs had higher densities than those without. Uncircumcised individuals with history of ulcerative STIs maybe more susceptible to acquisition and transmission of HIV through the prepuce than those without such history due to greater LCs densities.

Key words: Human Immunodeficiency Virus (HIV), Acquired Immunodeficiency Syndrome (AIDS), Langerhans Cells (LC), Sexually Transmitted Infections (STI).

\section{INTRODUCTION}

Macrophages have been considered to be the principal antigen presenting cells (APC) in the immune system. Compared to dendritic cells, macrophages are more abundant, uniformly distributed throughout the body, and are known to have antigen presenting capabilities. In epidermis, Langerhans cells function as sentinel antigen-presenting cells that can capture invading viruses such as Herpes Simplex Virus (HSV), Varicella-zoster virus (VZV) and Human Immunodeficiency Virus (HIV). This interaction between LCs and viruses result in highly variable responses, depending on the virus. For example, HSV induces apoptosis in LCs but HIV does not. LCs seem to be the first in a complex chain of antigen presentation to $T$ cells in lymph nodes for HSV and possibly VZV, or they transport virus to $T$ cells, as described for HIV and maybe VZV (Cunningham et al 2010).

Langerhans cells are immunoactive cells found in the skin and certain mucous membrane surfaces, including the foreskin and vagina. Recent research on human tissue samples has shown that these cells produce a protein called Langerin that helps to scavenge viruses from the environment and transport them elsewhere in the cell for destruction documented. While other authors have claimed that Langerhans cells in the foreskin are a portal for HIV infection,(McCoombe et al 2006) and have used this claim to argue for 
circumcision as a way to prevent HIV (Soilleux et al 2004).Others concludes in contrast that Langerin is a natural barrier to HIV-1 infection, and strategies to combat infection must enhance, preserve or, at the very least, not interfere with Langerin expression and function like the vagina, the foreskin has its own internal ecology of bacterial flora ( De witte et al 2007).

The densities of Langerhans cells have remained an area of scientific interests. A lot of investments have been made in doing circumcision to reduce HIV transmission by reducing Langerhans cells which have been implicated as antigen presenting cells. The current study examined the distribution and densities of Langerhans cells in the prepuce of adults and neonates and associated with age and STIs history at UTH and urban clinics in the Lusaka province of Zambia.

CD1a monoclonal antibody was used to identify the Langerhans cells.CD1a a nonpolymorphic MHC class 1 related cell surface glycoprotein expressed in association with beta 2 microglobulin.It is expressed by Langerhans cells, cortical thymocytes, dendritic cells, some malignancies of $T$ cells lineage like leukaemia, histocytosis $\mathrm{x}$ and various tissues.

\section{METHODOLOGY}

Samples were collected from consenting individuals who came for circumcision at UTH and Lusaka urban health centres. Prepuces from five males with history of ulcerative STI, ten without history of STI and five neonates were used in this study. Circumcision was provided by qualified and registered service providers using a dorsal slit method. They provided the specimen to the principal investigator. The specimens were fixed using $10 \%$ buffered formalin and transported to the histopathology laboratory where the tissues were grossed examined and embedded using paraffin wax. The formalin fixed paraffin blocks were then sectioned into 3-5um sections and then followed by immunohistochemistry staining. The primary

\section{RESULTS}

A total of 20 patients were sampled from amongst the clients who visited UTH and urban clinic for circumcision during the period of study. There were 5 neonates and 15 adults. Five adults had a history of STIS. Langerhans cell densities means with the participants with history of STIs having higher mean (88.4 \pm 7.092 ) with significant P-Value (0.001) than those without such history (69.4 \pm 8.847$)$ [Table 1]. Langerhans cell densities means with age, adults had higher mean with significant P-Value than neonates (Table 2). monoclonal antibodies (anti-CD1a) targeting Langerhans cells were used and LCs counting was done. The data collected was entered and stored into the data editor of IBM ${ }^{\circledR}$ SPSS $^{\circledR}$ and statistically analysed using IBM SPSS Statistics for Windows Version 20.0 (IBM Corp. Armonk, NY, USA). The dependent variable was Langerhans cells density. The Student's Independent Samples T-test was used to determine whether there were significant differences in the Langerhans cells' densities of the penile foreskins by comparing the means of each independent variable. If the P-value obtained was less than 0.05 , then the association was statistically significant at a confidence interval of $95 \%$.
The keratin thickness and density of Langerhans' cells were quantified. The results were that there was more keratin in the inner prepuce in men than boys. In both groups keratin was more in inner prepuce than outer surface if there was history of UTI. There was a higher density of Langerhans cells in boys than men, and boys with history of UTI had more Langerhans cells in the inner prepuce than those without the infection history. 
Anatomy Journal of Africa. 2018. Vol 7 (2): 1220 - 1223.

Table 1: Relationship between Langerhans cell density and STI status

\begin{tabular}{|l|l|l|l|l|l|}
\hline & Participants STI status & N & Mean & Standard deviation & P-Value \\
\hline \multirow{2}{*}{$\begin{array}{l}\text { Langerhans cell } \\
\text { densities (per } \mathrm{mm}^{2} \text { ) }\end{array}$} & None & 10 & 69.4 & 8.847 & 0.001 \\
\cline { 2 - 5 } & Present & 5 & 88.4 & 7.092 & \\
\hline
\end{tabular}

Table 2: Relationship between Langerhans cell density and age

\begin{tabular}{|l|l|l|l|l|l|}
\hline & Participants age & N & Mean & Standard deviation & P-Value \\
\hline \multirow{2}{*}{$\begin{array}{l}\text { Langerhans cell } \\
\text { densities (per mm }{ }^{2} \text { ) }\end{array}$} & Neonate & 5 & 36.6 & 5.273 & 0.000 \\
\cline { 2 - 5 } & Adults without STI Hx & 10 & 69.4 & 8.847 & \\
\cline { 2 - 5 } & Adults with STI Hx & 5 & 88.4 & 7.092 & \\
\hline
\end{tabular}

\section{DISCUSSION}

Based on this study, it can be inferred that uncircumcised individuals with history of ulcerative STIs may be susceptible to acquisition of HIV via the penile prepuce than uncircumcised individuals without history of ulcerative STIs due to their abundant Langerhans cells in the prepuce. The mean density in neonates Langerhans cells was found to be $36.6 \pm 5.272 / \mathrm{mm} 2$. The mean density of Langerhans cells in adults without past history of ulcerative STIs was $69.4 \pm 8.847 / \mathrm{mm} 2$ while those with history of ulcerative STIs was $88.4 \pm 7.092 / \mathrm{mm} 2$. These statistics for the Langerhans cells were all significant with a P-value $<0.05$ and confidence interval of $95 \%$.

Langerhans cell densities means of participants with history of STIs had higher means (88.4 \pm 7.092$)$ with significant P-Value (0.001) than those without history of STIs who had mean average of $69.4 \pm 8.847$. This is consistent with previous studies by Landay et al 2002 in which six adults with history of STIs had results indicating more Langerhans cells, CD4+ and macrophages than the eight children without history of STIs. A study by Qin et al in 2009 concluded that there was a higher density of Langerhans cells density in boys than men and went on further to show that boys with history of UTI had more Langerhans cells in the inner prepuce than those without such infection history. The mean density of Langerhans cells in foreskins of participants without a history of ulcerative STIs was $69.4 \pm 8.847$ which is comparable with the study by McCoombe et al 2006 which was 61cells/mm2.

The mean Langerhans cell densities when analysed for age showed that adults without STIs had higher mean average of $69.4 \pm$ 8.847 with significant $\mathrm{P}$-Value than neonates who had mean average of $36.6 \pm 5.273$. The result were different from Qin et al 2009 were six preschool boys and twenty healthy men without UTI history following male circumcisions were included in a study in china.. The study by Landay and Qin agree with our findings that injury of prepuce by STIs stimulates mobilization of Langerhans cells to the area to combat infectious bacteria, hence higher Langerhans cells densities in participants with history of STIs than those without STIs. Further Langerhans cells increase with age.

This study demonstrated a mobilization of Langerhans cells to inflamed prepuce. Ulcerative STIs cause local tissue inflammation migration, maturation and function of Langerhans cells in prepuce to combat infection and remain resident even after infection has cleared. It is this increase in Langerhans cells in the prepuce which has been implicated in higher rates of HIV transmission among people with prior STIs necessating $\mathrm{WHO}$ to recommend male circumcision.

In conclusion both age and past history of ulcerative STIs have an influence on the 
density of Langerhans cells on the penile prepuce. The neonates tend to have lower density on Langerhans cells compared to adults. Adults with history of ulcerative STIs had a greater densities of Langerhans cells compared to those who had no history of ulcerative STIs.

ACKNOWLEDGEMENTS: The authors would like to thank the study participants and the research assistants for their cooperation and trust in this research. We remain deeply indented to the University of Zambia through the Staff Development Office (SDO) and School of Medicine for the financial support rendered to us in sponsoring this study.

COMPETING INTERESTS: The authors declare no conflict of interest.

\section{REFERENCES}

1. Cunningham AL, Abendroth A, Jones C, Nasr N, Turville S. 2010. Viruses and Langerhans cells. Immunology and cell biology.88. 416-423. 10.1038/icb.42

2. Lot de Witte, Alexey N. 2007. Langerin is a natural barrier to HIV-1 transmission by Langerhans cells. Natural Medicine 13,367-371.

3. McCoombe SG, Short RV.2006. Potential HIV-1 target cells in the human penis. Aids 20(11): 1491-5.

4. Qin O, Zheng, Wang Y, Shen H F, Sun F, Ding W.2009. Langerhans cell density and degree of keratinisation in foreskins of Chinese preschool boys and male adults. International urology and nephrology. vol 41,issue 4 pp. 747-7535)

5. Soilleux EJ, Coleman N.2004. Expression of DC-SIGN in human foreskin may facilitate sexual transmission of HIV. J Clin Pathol 57:77-78

6. Weiss HA, Quigley MA, Hayes RJ.2000. Male circumcision and risk of HIV infection in subSaharan Africa: a systematic review and meta-analysis. AIDS 14:2361-2370.

7. Weiss GN, Sanders M, Westbrook KC.1993. The distribution and density of Langerhans cells in the human prepuce: site of a diminished immune response? J Med Sci 29: 42-43 